\title{
O uso de Metodologias Ativas na Formação Docente de Estudantes Residentes do curso de Letras - Língua portuguesa e Libras da UFRN
}

\author{
Everton da Silva Brito e Flávia Roldan Viana \\ Instituto Metrópole Digital - Universidade Federal do Rio Grande do Norte (UFRN) \\ Av. Sen. Salgado Filho, 3000 - Lagoa Nova, CEP: 59.078-970 - Natal - RN - Brasil \\ \{evertondsb36, flaviarviana.ufrn@gmail.com\}
}

\begin{abstract}
The incorporation of active methodologies supported by the use of digital instruments has been one of the major problems inherent in teacher education for basic education. One of the parallel challenges to this is the use of active methods with viable inclusion and teaching learning of deaf students. In this context, the work aims to present an experience report with resident students of the Portuguese Language and Libras course participants entitled "Active Methodologies in Deaf Education". The methodology used was the action research with qualitative approach. The results indicate the use of digital resources and active methods as facilitating tools in the teaching and learning of deaf students.
\end{abstract}

Resumo. A incorporação de metodologias ativas apoiadas pelo uso de instrumentos digitais, tem sido um dos grandes problemas inerentes a formação de professores para a educação básica. Um dos desafios paralelos a isso, é o uso de métodos ativos com víeis de inclusão e ensino aprendizagem de alunos surdos. Neste contexto, o trabalho tem como objetivo apresentar um relato de experiência com alunos residentes do curso Língua portuguesa e Libras participantes do Atelier intitulado "Metodologias Ativas na Educação de Surdos”. A metodologia utilizada foi a pesquisa-ação com abordagem qualitativa. Os resultados indicam o uso de recursos digitais e métodos ativos como instrumentos facilitadores no ensino aprendizagem de alunos surdos.

\section{Introdução}

A Educação de surdos é uma área do conhecimento situada no campo da Educação Especial e que se ocupa com estudos e investigações do processo de ensino e da aprendizagem de estudantes surdos, desde a Educação Infantil até o Ensino Superior. A Educação de surdos vem sendo discutida historicamente, ao longo dos anos, por dois grandes modelos: o modelo clínico-terapêutica (modelo médico, cujo foco central é a incapacidade do indivíduo) e o modelo socioantropológico (modelo social, cujas discussões partem da perspectiva da diferença), a partir dos conceitos de surdez e surdo.

Nesse âmbito a política de inclusão não deve se relacionar simplesmente à presença do aluno na sala regular, é preciso investir na estrutura da educação, desde a 
VIII Congresso Brasileiro de Informática na Educação (CBIE 2019)

Anais dos Workshops do VIII Congresso Brasileiro de Informática na Educação (WCBIE 2019)

formação dos profissionais até a prática pedagógica, para se propiciar o ambiente adequado e apto ao desenvolvimento e formação social do sujeito.

Segundo Antunes e Barroso (2015), o uso da tecnologia como ferramenta de ensino pode auxiliar no processo educacional, tanto para alunos, professores e gestores. Contudo, as consequências (positivas e negativas) provenientes da aplicação da tecnologia no âmbito educacional dependerá de seu uso perante as rotinas de trabalho.

Nesse contexto, as tecnologias digitais têm propiciado transformações em todos os aspectos da sociedade e principalmente nas formas de se relacionar e apreender novas habilidades. A escola como agente de reflexo das demandas sociais, precisa cada vez mais apropriar-se desses recursos e inserir nas novas dinâmicas de aprendizagem da sociedade tecnológica.

Essas novas dinâmicas devem pressupor o uso de metodologias ativas de ensino que dão ênfase ao papel protagonista do aprendiz, ao seu envolvimento direto, participativo e reflexivo em todas as etapas do processo, experimentando, desenhando, criando, com a orientação do professor utilizadas na estruturação metodológica e sequenciamento didático da prática educacional desenvolvida [Bacich; Moran 2017].

É com base nesse entendimento acerca da necessidade do investimento em práticas que proporcionem a inclusão do estudante surdo permeadas pelo uso de metodologias ativas com recursos digitais, que se fundamenta a proposta geral de apresentação deste trabalho.

Neste contexto, o presente trabalho tem como objetivo apresentar um relato de experiência envolvendo um Atelier pedagógico e formativo intitulado " Metodologias Ativas na Educação de Alunos Surdos" ofertado para alunos residentes do Curso de Licenciatura Letras- Língua portuguesa e Libras da Universidade Federal do Rio Grande

O Atelier seguiu o aspecto sugerido por Beira e Nakamoto (2016). Eles sugerem que a formação docente deve proporcionar condições necessárias para que os professores dominem tais ferramentas, superando abordagens triviais de metodologias ativas de ensino e do conhecimento sistemático dos processos de ensino e aprendizagem. $\mathrm{Na}$ oficina proposta, os participantes consideraram significativa a aprendizagem colaborativa com uso das tecnologias propostas.

O trabalho está organizado da seguinte forma: na Seção 2 são apresentados estudos relacionados a utilização de recursos digitais e metodologias ativas e legislação correlata a educação de surdos; a Seção 3 relaciona os objetivos da prática educativa desenvolvida; a Seção 4 apresenta a metodologia utilizada no desenvolvimento do trabalho; a Seção 5 apresenta os resultados obtidos durante a realização do Atelier; por fim, a Seção 6 detalha as Considerações Finais.

\section{Metodologia Ativa na educação de surdos}

Ao longo de décadas a educação tem apontado para a necessidade de práticas que busquem a igualdade e inclusão de todas as pessoas, sobretudo aquelas com alguma deficiência. Nesse enfoque, destacamos a Declaração Universal dos Direitos Humanos 
VIII Congresso Brasileiro de Informática na Educação (CBIE 2019)

Anais dos Workshops do VIII Congresso Brasileiro de Informática na Educação (WCBIE 2019)

(1948); a Conferência de Jomtien, que gerou a Declaração Mundial Sobre Educação Para Todos (1990); a Declaração de Salamanca (1994), que direcionou os debates em escala global acerca do tema; e, no Brasil, a Lei de Diretrizes e Bases da Educação (LDB), $n^{\circ}$ 9.394/96, que em seu capítulo V trata da educação especial e a Lei $\mathrm{n}^{\circ} 13.146$, de 6 de julho de 2015, que institui a Lei Brasileira de Inclusão da Pessoa com Deficiência (Estatuto da Pessoa com Deficiência).

O decreto 5.626, de 22 de dezembro de 2005 é o documento que regulamenta a Libras como língua oficial e primária na educação de surdos, além de tornar obrigatório a inserção da disciplina Libras nos cursos de formação de professores. A comunicação em Libras é rica de elementos visuais, de onde surge a cultura surda representada pelos sinais, pelo modo de ser, de expressar e conhecer o mundo [Perlin e Miranda 2003].

Dessa forma, entende-se que a língua de sinais utilizada pela comunidade surda é baseada principalmente no visual e gestual, portanto, as ferramentas a serem utilizadas não podem fugir disso. A utilização dos recursos visuais é comprovadamente uma boa estratégia para o professor que não tem domínio da língua de sinais [Morais, 2008].

Nessa perspectiva, e com base no ponto de vista dos próprios surdos o uso de mídias digitais constrói uma ponte com novas possibilidades de comunicação. As inovações oferecem um mundo visualmente fantástico para eles e são essas as principais ferramentas que trazem a perspectiva de profunda mudança nos usos e costumes [Rosa e Cruz, 2009].

Assim, diante desse cenário evidencia-se a importância e os impactos que o uso de recursos visuais pode trazer para a educação de surdos. Desse modo, as mídias surgem como recursos aliados e fundamentais para a inovação de novas práticas de ensino e aprendizagem nos ambientes escolares que atendam demandas de estudantes surdos.

Dentro desse contexto evidencia-se a importância de práticas pedagógicas voltadas para a inclusão e direcionadas ao propósito de construir uma sociedade justa e com mais igualdade., partindo-se de uma integração educativa que possibilite e brinde a sociedade e a cada indivíduo, de integrar-se nela com iguais direitos, mesmo com possibilidades diferentes, sustentadas em uma escola para todos com igualdade em tudo [Stobäus e Mosqueira 2004].

Nesse enfoque as metodologias ativas devem ser compreendidas como estratégias de ensino com foco no aluno, na personalização e no uso de modelos híbridos com aporte tecnológico, com destaque para alguns exemplos de modelos, como exemplo temos: a sala de aula invertida; a aprendizagem baseada em projetos; a aprendizagem por pares; a aprendizagem por times; a aprendizagem com jogos; a aprendizagem compartilhada e o estudo de caso, além de outras variações de modelos, que são pautados na aprendizagem por meio de objetivos individualizados, do grupo, ou pela construção de ambientes mediados por professores e tutores. [Moran, 2018].

As metodologias ativas possibilitam a potencialização das práticas didáticas nos diversos ambientes e níveis formativos, com vistas ao atendimento dos objetivos pedagógicos propostos. 
VIII Congresso Brasileiro de Informática na Educação (CBIE 2019)

Anais dos Workshops do VIII Congresso Brasileiro de Informática na Educação (WCBIE 2019)

Diante de todas essas potencialidades apresentadas e inerentes ao uso de métodos ativos, percebe-se o valor, os impactos, e a importância de que tais métodos possuem para promover transformações reais nas formas de ensinar e aprender. Esses são fatores primordiais para a produção do conhecimento em uma sociedade que dispõe de inúmeras formas e recursos tecnológicos que dinamizam o acesso à informação.

Neste sentido, o Atelier proposto buscou alinhar conhecimento didático, técnico e tecnológico, a fim de proporcionar mudanças nas práticas pedagógicas dos participantes, evidenciadas pela maior utilização de ferramentas digitais e práticas pedagógicas colaborativas, em sintonia com metodologias ativas de ensino, voltadas para o ensino do aluno surdo.

\section{Objetivos}

\subsection{Objetivo geral}

Apresentar as potencialidades do uso de metodologias ativas como recurso de ensino e aprendizagem para alunos surdos.

\subsection{Objetivos específicos}

- Instigar a realização de práticas didáticas com o uso de Metodologias Ativas no processo de ensino e aprendizagem de alunos surdos;

- Desenvolver com os estudantes residentes, práticas de ensino tomando como base o uso de ferramentas digitais que auxiliem na prática pedagógica dos estudantes residentes do curso de Letras Libras da UFRN;

- Analisar a percepção docente dos estudantes residentes do curso de Letras/Libras da UFRN acerca de suas práticas de ensino com estudantes surdos incluídos quanto às possibilidades do uso das metodologias ativas.

\section{Metodologia}

Utilizou-se como metodologia para desenvolvimento dos trabalhos a pesquisa-ação. Delimitou-se o problema da pesquisa relacionando-o ao uso de ferramentas digitais em práticas pedagógicas voltadas para o ensino e aprendizagem de alunos surdos, e identificação do nível de conhecimento acerca dos recursos digitais a serem trabalhados com os participantes do atelier. Promoveu-se uma ação perante os participantes e foram analisados os resultados desta ação.

Segundo Thiollent (2011) a pesquisa-ação é um tipo de pesquisa social de base empírica, idealizada e conduzida em estreita relação com uma ação ou resolução de problemas dentro de uma atividade em que os pesquisadores e os participantes estão envolvidos em modo cooperativo ou participativo, neste sentido, o planejamento do Atelier seguiu as etapas detalhadas no quadro 1. 
VIII Congresso Brasileiro de Informática na Educação (CBIE 2019)

Anais dos Workshops do VIII Congresso Brasileiro de Informática na Educação (WCBIE 2019)

O Atelier foi dividido inicialmente em duas etapas, com duração de 4 horas em cada. No primeiro encontro foi apresentado aos participantes as ações e objetivos que norteariam todo o processo. Em seguida iniciamos a discussão teórica acerca dos principais tipos e características das metodologias ativas e a evolução da educação 1.0 até a educação 3.0, possibilitando-se a reflexão, análise dos impactos, transformações e novas formas de ensinar e aprender. Por fim, destacamos algumas ferramentas (Plickers; Mentimenter; Google Docs; Google Forms; Kahoot e Google sala de aula;), tecnológicas de auxílio para a produção de aulas com foco na aprendizagem ativa do aluno.

No segundo encontro, os participantes foram divididos em grupos de livre escolha para que pudessem refletir e planejar ações didático pedagógicas de intervenção com o uso de pelo menos uma metodologia ativa, dentre as apresentadas no primeiro encontro. Em seguida todos os grupos apresentaram suas propostas, a fim de verificarmos a pertinência, os objetivos, hipóteses e validar para uma aplicação eficiente.

\begin{tabular}{|c|c|}
\hline Fase & Descrição \\
\hline $\begin{array}{l}\text { Levantamento } \\
\text { bibliográfico }\end{array}$ & $\begin{array}{l}\text { Levantamento bibliográfico relacionado aos principais conceitos da Escola } 3.0 \text { e o Perfil do } \\
\text { Educador } 3.0 \text {; modelos de aprendizagem de alunos surdos; conceituação de metodologias } \\
\text { ativas e recursos digitais }\end{array}$ \\
\hline $\begin{array}{l}\text { Planejamento } \\
\text { do Atelier }\end{array}$ & $\begin{array}{l}\text { Foram definidos o objetivo geral, conteúdos, metodologia, recursos necessários, } \\
\text { instrumentos de avaliação e resultados esperados com o atelier; bem como elaboração das } \\
\text { atividades relacionadas ao aprendizado das ferramentas digitais: 1- Mentimeter, } 2 \text { - Google } \\
\text { Forms, } 3 \text { - Google Docs, } 4 \text { - Coggle, } 5 \text { - Plickers e } 6 \text { - kahoot. }\end{array}$ \\
\hline $\begin{array}{l}\text { Diagnóstico } \\
\text { inicial }\end{array}$ & $\begin{array}{l}\text { Utilizou-se um questionário para colher informações inerentes ao diagnóstico do perfil dos } \\
\text { participantes do Atelier "Metodologia ativa na educação de surdos ", bem como identificar } \\
\text { o nível de conhecimento destes nas seguintes temáticas: } 1 \text { - Ferramentas interativas de apoio } \\
\text { a práticas pedagógicas digitais, } 2 \text { - Metodologias Ativas de Ensino, } 3 \text { - Principais habilidades } \\
\text { para os profissionais do futuro, } 4 \text { - Escola } 3.0 \text { e o Perfil do Educador } 3.0 \text { e } 5 \text { - Benefícios da } \\
\text { utilização de ambientes e sistemas digitais para a educação". }\end{array}$ \\
\hline $\begin{array}{l}\text { Execução do } \\
\text { Atelier }\end{array}$ & $\begin{array}{l}\text { A execução do Atelier teve como objetivo permitir aos participantes conhecer ferramentas } \\
\text { digitais de apoio a práticas educacionais com metodologias ativas e recursos digitais, } \\
\text { produção de aulas ativas, bem como reflexão sobre as novas formas de ensinar e aprender. }\end{array}$ \\
\hline $\begin{array}{l}\text { Avaliação de } \\
\text { reação }\end{array}$ & $\begin{array}{l}\text { Ao final do Atelier foi aplicado um novo questionário para avaliação do Atelier } \\
\text { "Metodologia ativa na educação de surdos" por parte dos participantes, bem como avaliar } \\
\text { a mudança de sua percepção em relação aos aspectos avaliados no Diagnóstico inicial. Os } \\
\text { resultados obtidos foram analisados. }\end{array}$ \\
\hline
\end{tabular}

Quadro 1. Planejamento do Atelier 
VIII Congresso Brasileiro de Informática na Educação (CBIE 2019)

Anais dos Workshops do VIII Congresso Brasileiro de Informática na Educação (WCBIE 2019)

\section{Resultados e Discussões}

Nesta seção, iremos discutir os dados coletados durante a avaliação de reação realizada com os residentes participantes dos dois encontros do Atelier formativo, "Metodologias Ativas na educação de surdos", incluindo sua percepção quanto ao caráter inovador da proposta; importância na formação docente; conhecimento dos recursos e conceitos, além da observação acerca dos impactos positivos para a prática didática com alunos surdos.

Na Figura 1 é ilustrado o conhecimento e uso de metodologias ativas em sala de aula pelos participantes do Atelier. Identificamos que apenas $36,1 \%$ já utilizaram métodos ativos nas aulas, enquanto que $63,9 \%$ nunca usaram, ou desconheciam totalmente os $\mathrm{s}$ conceitos balizadores da pedagogia ativa.

\section{- Você conhece e/ou já utilizou alguma metodologia ativa em suas aulas?}

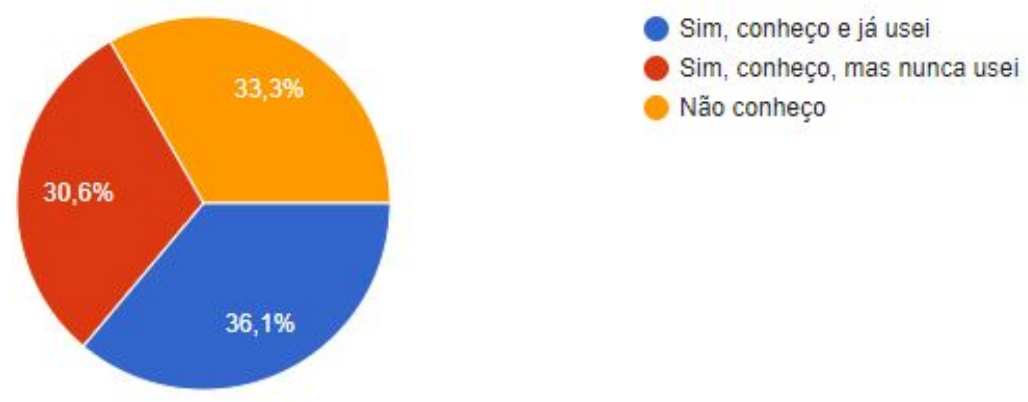

Figura 1: Conhecimento acerca do uso de metodologias ativas

Outro aspecto avaliado na fase de diagnóstico foi acerca da regularidade com que os participantes do Atelier elaboram os planejamentos de aulas, com foco no uso de recursos tecnológicos. Uma vez que um dos propósitos do Atelier foi oportunizar o conhecimento de recursos digitais de apoio às práticas pedagógicas ativas para o ensino aprendizagem do aluno surdo. Neste sentido, 25\% dos participantes afirmaram que sempre planejam o uso de tecnologia nas aulas, enquanto que $38,9 \%$ raramente pensa no recurso para uso em suas aulas, ver Figura 2. 
VIII Congresso Brasileiro de Informática na Educação (CBIE 2019)

Anais dos Workshops do VIII Congresso Brasileiro de Informática na Educação (WCBIE 2019)

Com qual regularidade você pensa/planeja o uso de tecnologias em sala de aula?

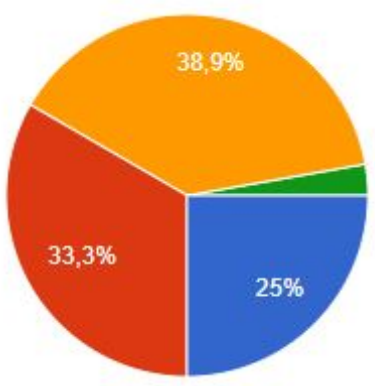

Figura 2: Regularidade do uso de tecnologias em sala de aula

Ao final do Atelier foi realizada uma avaliação de reação através de um novo questionário que teve como objetivo principal colher informações, acerca de possíveis mudanças na percepção dos participantes, quanto a importância de realizar pratica com foco nas metodologias ativas e uso de recursos tecnológicos, ver figuras 3 e 4 .

Como você avaliaria a sua vontade de mudar suas atitudes, baseado no que aprendeu durante o Atelier?

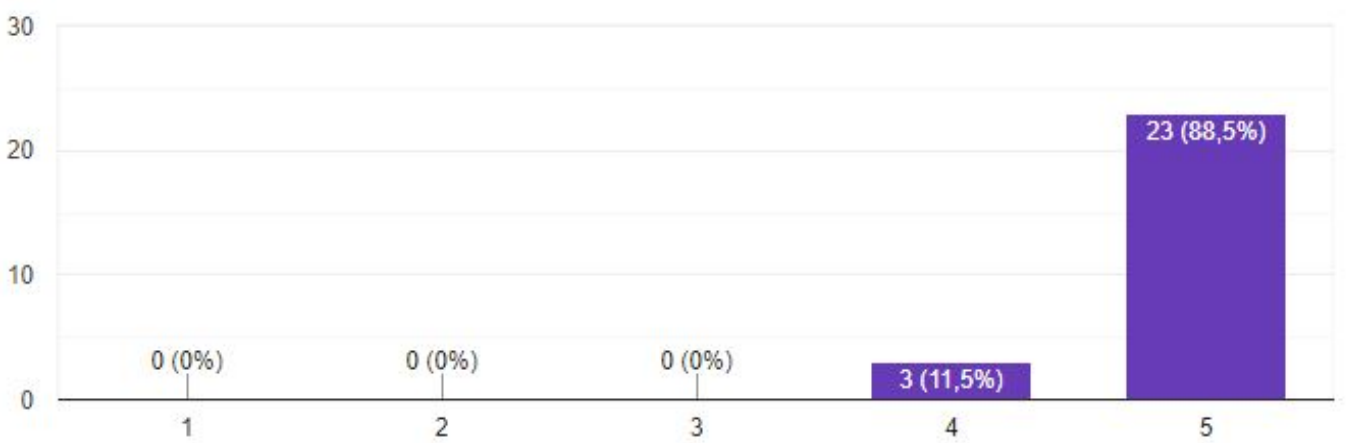

Figura 3: Avaliação de mudança das práticas didáticas 
VIII Congresso Brasileiro de Informática na Educação (CBIE 2019)

Anais dos Workshops do VIII Congresso Brasileiro de Informática na Educação (WCBIE 2019)

Como você avalia a importância das metodologias ativas de Ensino que colocam o estudante no centro dos processos de ensino e aprendizagem?

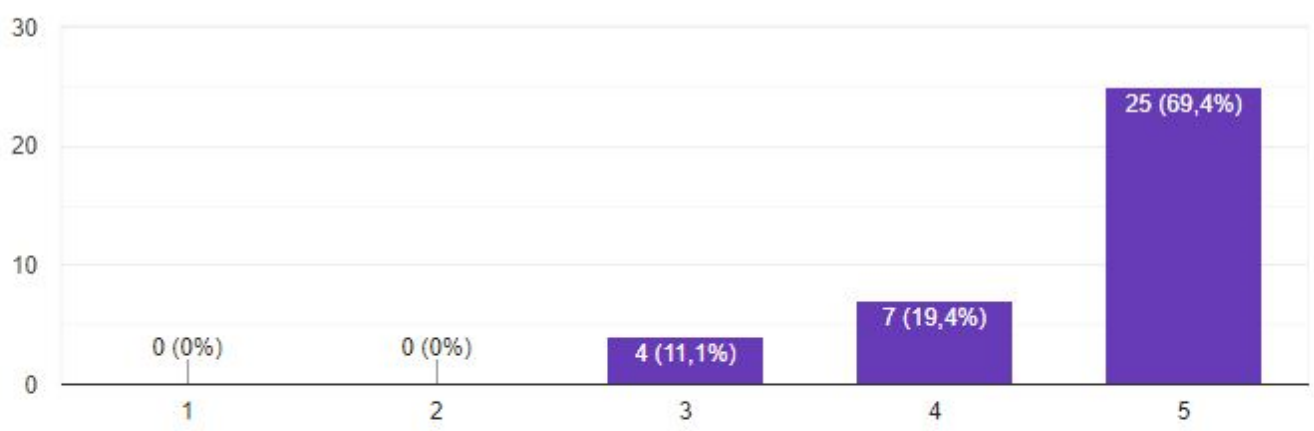

Figura 4: Importância do uso de metodologias ativas

\section{Considerações Finais}

Considera-se que a atividade de formação ministrada em formato de Atelier intitulado "Metodologias Ativas na educação de Surdos" atingiu o objetivo de apresentar recursos digitais no apoio a práticas voltadas para a educação de surdos, bem como proporcionar mudanças na percepção dos educadores alvo da formação, no que diz respeito aos benefícios das práticas educacionais inovadoras com a utilização de recursos digitais em sala de aula com surdos incluídos.

As práticas pedagógicas desenvolvidas em conjunto com recursos digitais apresentadas foram classificadas como recursos pedagógicos importantes pelos participantes do Atelier, principalmente pela possibilidade de conciliar com metodologias ativas de Ensino, a exemplo do Design Thinking pelas ferramentas Mentimeter e Coggle; Sala de Aula Invertida e Aprendizagem Colaborativa com auxílio do Google Forms e Docs; Aprendizagem por Pares e TBL com Plickers; Gamificação com Kahoot.

\section{Referências}

Antunes, M. A. e Barroso, F. (2015) “Tecnologias na Educação: Ferramentas Digitais Facilitadores da Prática Docente", Pesquisa e Debate em Educação, v.5, p.124-131.

Bacich, Lillian; Moran, José. (2017) "Metodologias Ativas para uma Educação Inovadora: Uma Abordagem Teórico-Prática. Porto Alegre: Penso Editora.

Beira, D. G. e Nakamoto, Paula Teixeira. A Formação docente inicial e continuada prepara os Professores para o Uso das Tecnologias de Informação e Comunicação (TICs) em sala de aula?. In: Anais do XXII Workshop de Informática na Escola (WIE 2016), p. 825-834. DOI:10.5753/cbie.wie.2016.825. 
VIII Congresso Brasileiro de Informática na Educação (CBIE 2019)

Anais dos Workshops do VIII Congresso Brasileiro de Informática na Educação (WCBIE 2019)

Brasil. "Lei de Diretrizes e Bases da Educação Nacional". Presidência da República Casa Civil. Distrito Federal, DF, 26 dez, 1996. Disponível em: $<$ http://www.presidencia.gov.br/ccivil_03/Leis/L9394.htm>. Acesso em: 08 jun. 2019.

Conferência Mundial de Educação para Todos. "Declaração Mundial de Educação para Todos". Plano de Ação para Satisfazer as Necessidades Básicas de Aprendizagem. Brasília, DF: UNIFEC, 1990.

Declaração de Salamanca. "Sobre Princípios, Políticas e Práticas na Área das Necessidades Educativas Especiais”. 1994, Salamanca-Espanha.

Morais, Luciana Lima. de. (2008) "O ensino da Geografia com o enfoque na aprendizagem dos surdos: perspectivas e desafios." Revista Bibliocanto, Natal, v.5, n.1, jan./jun.. Disponível em: < https://periodicos.ufrn.br/bibliocanto/article/view/57>. Acesso em 27 set. 2017.

Moran, José. (2018) "Metodologias ativas para uma aprendizagem mais profunda". Metodologias ativas para uma educação inovadora: uma abordagem teórico-prática. Porto Alegre: Penso, p. 02-25.

Perlin Gladis; Miranda Wilson. (2003) "Surdos: o Narrar e a Política In Estudos Surdos" - Ponto de Vista: Revista de Educação e Processos Inclusivos no 5, UFSC/ NUP/CED, Florianópolis.

Rosa, Andréa da Silva; Cruz, Cristiano Cordeiro. (2001) "Internet: fator de inclusão da pessoa surda”. Revista Online da Biblioteca Prof. Joel Martins, v. 2, n. 3, p. 38-54. Disponível em: <http://www.brapci.ufpr.br/brapci/v/a/3083>. Acesso em: 27 set. 2017.

Stobäus, Claus D.; Mosqueira, Juan J. Mouriño. (2004) "Educação Especial: em direção à Educação Inclusiva”. 2. ed. Porto Alegre: EDIPUCRS.

Thiollent, M. (2011) “Metodologia da pesquisa-ação”, São Paulo: Cortez Editora. 